\title{
Multimodal Atomic Scale Characterization of Structural and Compositional Changes During Shear Deformation of Materials
}

\author{
Bharat Gwalani ${ }^{1}$, Elizabeth J. Kautz ${ }^{2}$, Tiffany Kaspar ${ }^{1}$, Libor Kovarik ${ }^{3}$, Vineet V. Joshi ${ }^{4}$, Suveen \\ Mathaudhu ${ }^{4,5}$, Aashish Rohatgi ${ }^{4}$, Peter Sushko ${ }^{1}$ and Arun Devaraj ${ }^{1^{*}}$ \\ ${ }^{1}$ Physical and Computational Sciences Directorate, Pacific Northwest National Laboratory, \\ Richland, WA \\ ${ }^{2}$ National Security Directorate, Pacific Northwest National Laboratory, Richland, WA \\ ${ }^{3}$ Environmental Molecular Sciences Laboratory, Pacific Northwest National Laboratory, \\ Richland, WA \\ ${ }^{4}$ Energy and Environment Directorate, Pacific Northwest National Laboratory Richland USA \\ ${ }^{5}$ Mechanical Engineering and Material Science and Engineering, University of California \\ Riverside, CA \\ *Corresponding author: arun.devaraj@pnnl.gov
}

Traditional alloy manufacturing has often relied on melt processing of elements, which predominantly limit the outcome to formation of equilibrium phases. A newly developed solid phase processing method named shear assisted processing and extrusion (ShAPE) enables us to achieve novel solid phase microstructural pathways during processing that result in unique texture, nanostructured precipitates and mechanical properties while fully avoiding melt processing routes [1-3]. The microstructural evolution during the ShAPE process is dictated by the atomic scale mass and energy transfer that occurs in materials when subjected to high shear-induced forces. The mechanisms involved during the ShAPE process can be directly related to atomic-scale as well as microstructural events during severe plastic deformation (SPD) processing of materials. High speed atomic relocation while shearing, with concurrent heating of the material, make the atomic scale mechanisms in such processes quite complex to understand. The complexity arises from the interaction of different process variables, such as rotational motion of the tool, and multidimensional nature of the shear forces. Additionally, the variability of friction forces and thus the in situ heating of the system, flow of material, and dynamic cooling during the process are congruently occurring interrelated phenomena which make it difficult to model [4]. Furthermore, the physical properties of the starting material (strength, ductility, miscibility, heat conductivity, work hardenability, melting point, ductile to brittle transition temperature) also directly impact the microstructural evolution pathway. Thus, it becomes extremely difficult to make a universal model for such complex processes without further experimental input towards understanding the dynamic events under high shear-assisted deformation, especially at near-atomic resolution.

To address this critical need, at PNNL we are developing systematic experimental approaches to decouple the effects of various factors and develop a comprehensive, fundamental atomic-scale understanding of SPD during ShAPE processing. We synthesize model materials with very well understood interfaces possessing unique structural and compositional features. Then we use a pinon-disk tribometer to emulate bulk solid phase processing (SPP) conditions in small material volumes, thus enabling us to analyze shear deformations at micro- to atomic length scales and provide mechanistic insight into the behavior of bulk material volumes under SPP conditions. The structural and compositional changes occurring at the interfaces before and after shear deformation are analyzed using secondary electron microscopy (SEM), aberration corrected transmission electron microscopy (TEM), and atom probe tomography (APT). 
The results from such detailed microstructural analysis of an as-cast AZ31-2wt\% Si alloy billet and a ShAPE processed pipe are shown in Figure 1. The Figure shows SEM, electron dispersive spectroscopy (EDS), and APT results from before and after ShAPE processing of the alloy. A nano-structuring of $\mathrm{Mg}_{2} \mathrm{Si}$ precipitates, likely due to dissolution and reprecipitation during processing, and shear-induced dissolution of $\mathrm{Zn}$ in the $\mathrm{Mg}$ matrix are observed.

\section{References:}

[1] S Mathaudhu, N Overman, S Whalen, M Olszta, D Catalini, K Kruska, J Darsell, V Joshi, X Jiang, A Devaraj, G Grant, Magnesium Technology 1, 65-67 (2019).

[2] Jens T Darsell, Nicole R Overman, Vineet V Joshi, Scott A Whalen, Suveen N Mathaudhu, Jens T Darsell, Nicole R Overman, Vineet V Joshi, Scott A Whalen, Suveen N Mathaudhu, J of Mat. Eng. and Performance, 27.8, 1-12 (2018)

[3] Martin Georges, Current Opinion in Solid State and Mat. Science 3.6, 552-557 (1998).
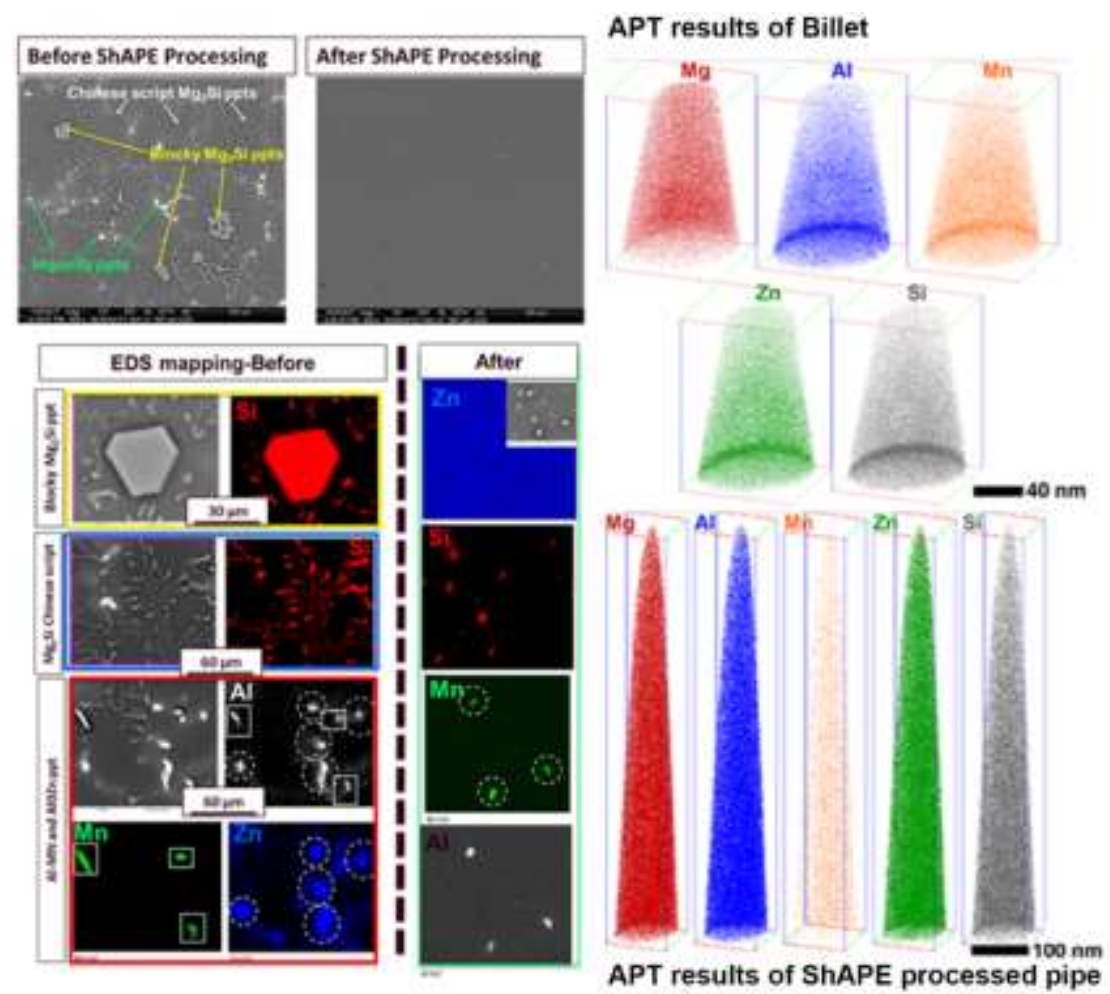

Figure 1. SEM and EDS characterization (left) of AZ31-2 wt.\% Si alloy before and after ShAPE processing. Dissolution and re-precipitation during processing leads to nano-structuring of the $\mathrm{Mg}_{2} \mathrm{Si}$ precipitates. APT characterization (right) of matrix AZ31-2 wt.\% Si alloy before (top) and after (bottom) ShAPE processing enables quantitative compositional analysis of minor alloying elements. 